\title{
Nutrition and brain aging: role of fatty acids with an epidemiological perspective
}

\section{Cécilia SAMIERI \\ Pascale BARBERGER-GATEAU}

Inserm, U897,

Équipe Épidémiologie de la nutrition et des comportements alimentaires, Université Bordeaux Segalen,

Case 11 ,

146 rue Leo-Saignat,

F-33076 Bordeaux cedex,

France

$<$ cecilia.samieri@isped.u-bordeaux2. fr $>$

\begin{abstract}
In the absence of identified etiologic treatment for dementia, the potential preventive role of nutrition may offer an interesting perspective. The objective of the thesis of C. Samieri was to study the association between nutrition and brain aging in 1,796 subjects, aged 65 y or older, from the Bordeaux sample of the Three-City study, with a particular emphasis on fatty acids. Considering the multidimensional nature of nutritional data, several complementary strategies were used. At the global diet level, dietary patterns actually observed in the population were identified by exploratory methods. Older subjects with a "healthy" pattern, who consumed more than 3.5 weekly servings of fish in men and more than 6 daily servings of fruits and vegetables in women, showed a better cognitive and psychological health. Adherence to the Mediterranean diet, measured according to a score-based confirmatory method, was associated with slower global cognitive decline after 5 y of follow-up. At the nutrient biomarker level, higher plasma eicosapentaenoic acid (EPA), a long-chain omega-3 fatty acid, was associated with a decreased dementia risk, and the omega-6-to-omega-3 fatty acids ratio to an increased risk, particularly in depressed subjects. EPA was also related to slower working memory decline in depressed subjects or in carriers of the $\varepsilon 4$ allele of the ApoE gene. Docosahexaenoic acid was related to slower working memory decline only in ApoE4 carriers. Overall, this work suggests a positive impact of a healthy diet rich in fruits and vegetables and fish, and notably the Mediterranean diet, on cognition in older subjects. Long-chain n-3 PUFA, in particular EPA, may be key protective nutrients against risk of dementia and cognitive decline.
\end{abstract}

Key words: diet, nutrition, cognition, aging, fatty acids, omega-3

diagnosed when the onset of cognitive impairment is progressive and insidious and when no other systemic or brain disease may account for the progressive cognitive deficits. AD specific brain lesions include extracellular accumulation of $\beta$-amyloid peptides and intracellular accumulation of hyperphosphorylated protein Tau. New research criteria have been recently proposed, combining evidence of $A D$ pathology based on neuroimaging and biological biomarkers, and clinical signs of memory impairment of the hippocampal type (episodic memory) (Dubois et al., 2007).

$\mathrm{AD}$ has been recently defined as a complex clinico-biological entity, characterized by a long-term pathological process (Dubois et al., 2010; Jack et al.,
2010). Indeed, while dementia diagnosis is made when cognitive impairment is widespread enough to generate disability, the first cognitive symptoms start more than 10 y before (Amieva et al., 2008), and the first brain lesions leading to neurodegeneration could occur decades before the clinical manifestations (Reiman et al., 2004). This long time-lag between the first lesions in the brain and the clinical symptoms defines a large window of opportunity for potential preventive factors, before irreversible neurodegeneration.

An important feature for the study of risk factors of brain aging is that late-life dementia is multifactorial. It results from $A D$ specific lesions and other lesions also found in normal aging such as vascular

To cite this article: Samieri C, Barberger-Gateau P. Nutrition and brain aging: role of fatty acids with an epidemiological perspective. OCL 2011; 18(4): 228-35. doi : 10.1684/ocl.2011.0397 
lesions, inflammation and oxidative stress, both balanced by compensatory mechanisms. Accordingly, a life-course etiologic model of dementia has been proposed, in which genetic risk factors may interact with environmental factors to modulate the risk of dementia and AD (Fotuhi et al., 2009). Age is the strongest known risk factor for $A D$, followed by genetic susceptibility, conferred by the $\varepsilon 4$ allele of the ApoE (ApoE\&4) gene, which increases the risk of AD by 3 times in Causasian heterozygotes and by 15 times in homozygotes (Farrer et al., 1997). Moreover, novel susceptibility genes for $A D$ have been recently identified (Sleegers et al., 2010). Among environmental risk factors, vascular risk factors such as hypertension, diabetes, high blood cholesterol or obesity at midlife, have been associated with higher risk of dementia or higher cognitive decline, leading to progressive evidence that cardiovascular diseases and dementia shared numerous common risk factors (Reitz et al., 2011). Nutritional factors, capable of modulating vascular risk factors and probably having a direct impact on brain function, have emerged as interesting candidates for prevention, especially longchain omega-3 polyunsaturated fatty acids (n-3 PUFA).

Indeed, long-chain n-3 PUFA may exert beneficial effects against brain aging, explained by their major structural and functional role in neurone membranes, vascular and anti-inflammatory properties as well as their potential ability to modulate neuro-inflammation and the expression of neuronal-plasticity related genes (see for review (Cunnane et al., 2009)). Several longitudinal epidemiological studies suggested a protective association between fish consumption, the main source of long-chain n-3 PUFA in humans, or eicosapentaenoic acid (EPA) and docosahexaenoic acid (DHA) consumption and cognitive decline or dementia risk. However, fewer studies have assessed the exposition to longchain n-3 PUFA by measuring blood circulating levels, which enable to take into account inter-individual variations in biodisponibility. Finally, intervention studies yielded mixed and mostly negative results (Cunnane et al., 2009). Thus, more research was needed to understand how long-chain n-3 PUFA would be biologically related to brain aging.
In addition, other foods and nutrients, such as fruits and vegetables rich in antioxidant compounds and folates, were evoked as potential preventive factors in brain aging (Feart et al., 2010). More generally, nutrients and foods are consumed in combination, so they could exert additive or synergistic effects on brain aging, which may not be captured in single-nutrient based studies (Moeller et al., 2007). Dietary patterns, which represent a combination of foods, may be better associated to disease risk than single foods or nutrients.

Dietary patterns have been classified into two main categories (Moeller et al., 2007). A priori dietary patterns are defined by scores, computed according to previous hypotheses on the beneficial or deleterious effects of nutritional factors on health. A priori dietary patterns are therefore classified under the category of confirmatory methods. In contrast, $a$ posteriori dietary patterns are defined either by scores derived from principal component analysis, or by specific clusters derived from cluster analysis, both strategies being undertaken without any prior hypothesis on the diet-disease relationship. Thus, a posteriori dietary patterns fit to the definition of exploratory methods.

Considering the multidimensional nature of nutritional data, an accurate study of the relationship between nutrition and brain aging must therefore include two complementary approaches to estimate nutritional exposure: a whole-diet approach (e.g global level) and a nutrient-based approach. The main objective of the thesis of C. Samieri summarized in this report was to study, with an epidemiological perspective (e.g. at the population (evel), the relationship between nutrition and brain aging in 1,796 subjects, aged 65 y or older, from the Bordeaux sample of the three-City (3C) study. A particular interest was given to n-3 PUFA. Thus, the first aim of the thesis was, using a whole-diet approach, to identify dietary patterns by exploratory analysis and to relate them to cognitive performances and depressive symptoms in older subjects. Moreover, adherence to a Mediterranean-type diet was assessed by an a priori score and was related to future cognitive decline and dementia risk. The second aim was, using a nutrient-based approach focused on long-chain $\mathrm{n}-3$ PUFA, to study the association between plasma fatty acids and future dementia risk and cognitive decline, taking into account the potential modifying role of depressive symptoms and the ApoE4 allele.

\section{The Three-City cohort study}

Cohort studies offer a unique opportunity to assess risk factors of dementia and $A D$ early in the course of the disease, before the clinical phase associated with widespread and irreversible brain damage. These analyses were conducted within the Bordeaux sample of the 3C study, a prospective cohort study of vascular risk factors for dementia which started in 1999-2000 and included 9,294 community dwellers in Bordeaux ( $n=2,104)$, Dijon $(n=4,931)$ and Montpellier $(n=2,259)$, France (Three-City Study Group, 2003). Baseline data collection included socio-demographic and lifestyle characteristics, symptoms and complaints, main chronic conditions, neuropsychological testing, physical examination and blood sampling, enabling to perform ApoE genotyping. Four follow-up examinations were performed 2, 4, 7 and 10 y after baseline.

At each visit, cognitive performance was assessed by trained psychologists using a battery of neuropsychological tests, including: the Mini Mental State Examination (MMSE), assessing global cognitive function; the Isaac's Set test (IST), assessing verbal fluency; the Benton Visual Retention Test (BVRT), assessing working memory; Trails Making tests part A (TMT-A) and B (TMT-B), assessing executive functioning; and the Free and Cued Selective Reminding Test (FCSRT), assessing episodic memory. Diagnosis of dementia was based on a three-step procedure. All potential prevalent and incident cases of dementia were investigated and reviewed by an independent committee of neurologists to obtain a consensus on the diagnosis and aetiology according to the criteria of the Diagnostic and Statistical Manual of Mental Disorders, fourth edition (American Psychiatric Association, 1994). At baseline, the intensity of depressive symptoms was assessed using the Center for Epidemiologic Studies Depression (CESD) scale, and high depressive symptoms were defined as a CESD score of 17 or more in men and 23 or more in women. 
Proportions of fatty acids were measured at baseline in total plasma in 1,518 subjects, and an extensive dietary survey, including a food frequency questionnaire and a $24 \mathrm{~h}$ dietary recall, was administered at the 2 y visit.

\section{Dietary patterns and brain aging}

\section{A posteriori dietary patterns and brain aging}

Actual dietary patterns as observed in a population of older subjects were identified by exploratory statistical methods (Samieri et al., 2008). Among 647 men and 1,033 women from the Bordeaux sample of the $3 \mathrm{C}$ study, cluster analysis was used on weekly servings of specific food groups to divide the subjects into homogeneous non-overlapping subgroups with a similar pattern of mean food intake. In order to stabilize the final partition, a mixed clustering method was applied. Five dietary clusters were identified in each gender (figures 1 and 2). In both male and female, the first cluster was characterized by a lower mean number of weekly servings that the overall population for all food groups and a lower total energy intake. It was thus labelled "small eaters". The second cluster, labelled "biscuits and snacking", was characterized by a high consumption of biscuits and cakes and included a high proportion of individual consuming frequent snacks. The third cluster was a "healthy" dietary pattern, mainly characterized by a high consumption of fish in men and of fruits and vegetables in women. The fourth cluster was characterized by a greater consumption of "charcuterie, meat and alcohol" in men and "charcuterie and starchy foods" in women. Finally, the fifth cluster included frequent "pasta eaters" in men and frequent "pizzas and sandwiches" eaters in women (Samieri et al., 2008).

Older subjects from the "healthy" pattern, who consumed more than 3.5 weekly servings of fish in men and more than 6 daily servings of fruits and vegetables in women, showed higher global cognitive performances as assessed by the score to the MMSE. Moreover, women from the "healthy pattern" had lower depressive symptoms as assessed by the CESD. Women in the "biscuits and snacking" cluster had greater risk of poor perceived health compared to "healthy" eaters. Overall, this work suggested a potential protective role for a "healthy" pattern against brain aging, although the cross-sectional nature of the design prevented to infer any causality in the observed associations. Moreover, these findings identified clusters of subjects with a particular food behaviour associated with poorer cognitive and psychological health, which may be targeted for nutrition policies in order to prevent brain aging.

\section{Adherence to a Mediterranean diet, cognitive decline and risk of} dementia

The Mediterranean diet (MeDi) is the traditional diet of the Greek and SouthItalian populations and is mainly featured by an important consumption of plant foods, fish, monounsaturated fats from olive oil as the main fat source, a moderate consumption of alcohol in the form of wine, and a low consumption of meat and dairy products. Adherence to the MeDi has been previously associated with a reduced risk for several chronic diseases (Sofi et al., 2010).

We investigated the relationship between adherence to the MeDi and cognitive decline and dementia risk in the $3 \mathrm{C}$ study (Feart et al., 2009). Among 1,410 participants from the Bordeaux sample of the $3 \mathrm{C}$ study, a score of adherence to the MeDi was constructed at the first follow-up, as originally described by Trichopoulou et al. (Trichopoulou et al., 1995) (see paper by Barberger-Gateau et al. in this volume).

Higher MeDi score was significantly associated with lower decline to MMSE performances over 5 y of follow-up ( $\beta=-$ $0.006, P=0.04$ for 1 point-increase of MeDi score), after adjustment for numerous potential confounders (age, sex, education, marital status energy intake, physical activity, depressive symptoms, medication use, ApoE4 genotype, cardiovascular risk factors and stroke). No association was found between MeDi score and the risk for incident dementia. However, due to a relatively small number of incident dementia cases $(n=99)$, the power to detect such association was limited in this study.

Suggesting that higher adherence to the MeDi lower decline to global cognitive performances, this result extended to the field of $A D$ the potential beneficial role of the MeDi already suggested in the prevention of other chronic diseases. Such a preventive role may rely on additional or synergistic action of several nutrients, according to biological mechanisms which remain to be determined.

\section{Long-chain n-3 PUFA and brain aging}

Long-chain n-3 PUFA EPA and DHA may be key nutrients involved in the beneficial role of the MeDi against cognitive decline (Feart et al., 2011) (see article by Barberger-Gateau et al. in this volume). The second part of the thesis was therefore targeted at the nutrient biomarker level, and consisted in assessing the relationship between long-chain $n-3$ PUFA measured in plasma and brain aging.

The potential confounding or modifying effect of depressive status in this relationship was examined. Indeed, depressive status was associated with blood levels of long-chain n-3 PUFA in several studies (Lin et al., 2010), and, notably, with plasma EPA levels in the $3 \mathrm{C}$ study (Feart et al., 2008). However, since the observed associations were all crosssectional, no causal relationship could be inferred from these studies: lower exposure to long-chain n-3 PUFA could cause depressive symptoms, or, alternatively, depression could change dietary habits and therefore lower fish consumption, leading to lower blood levels of long-chain n-3 PUFA. Moreover, several studies suggested that depression could be a potential risk factor for dementia (Panza et al., 2010). Thus, one could expect that a positive association between blood levels of long-chain $\mathrm{n}-3$ PUFA and depressive status explained the potential association between these fatty acids and dementia risk or cognitive decline (e.g confounding effect of depression). Otherwise, the presence of high depressive symptoms could enhance the effect of long-chain $\mathrm{n}-3$ PUFA on cognition (e.g modifying effect of depression, detected as an interaction in epidemiological studies).

\section{Plasma long-chain n-3 PUFA and risk of dementia}

First, the relationship between plasma fatty acids and dementia risk over $4 \mathrm{y}$ of follow-up was studied, taking into 


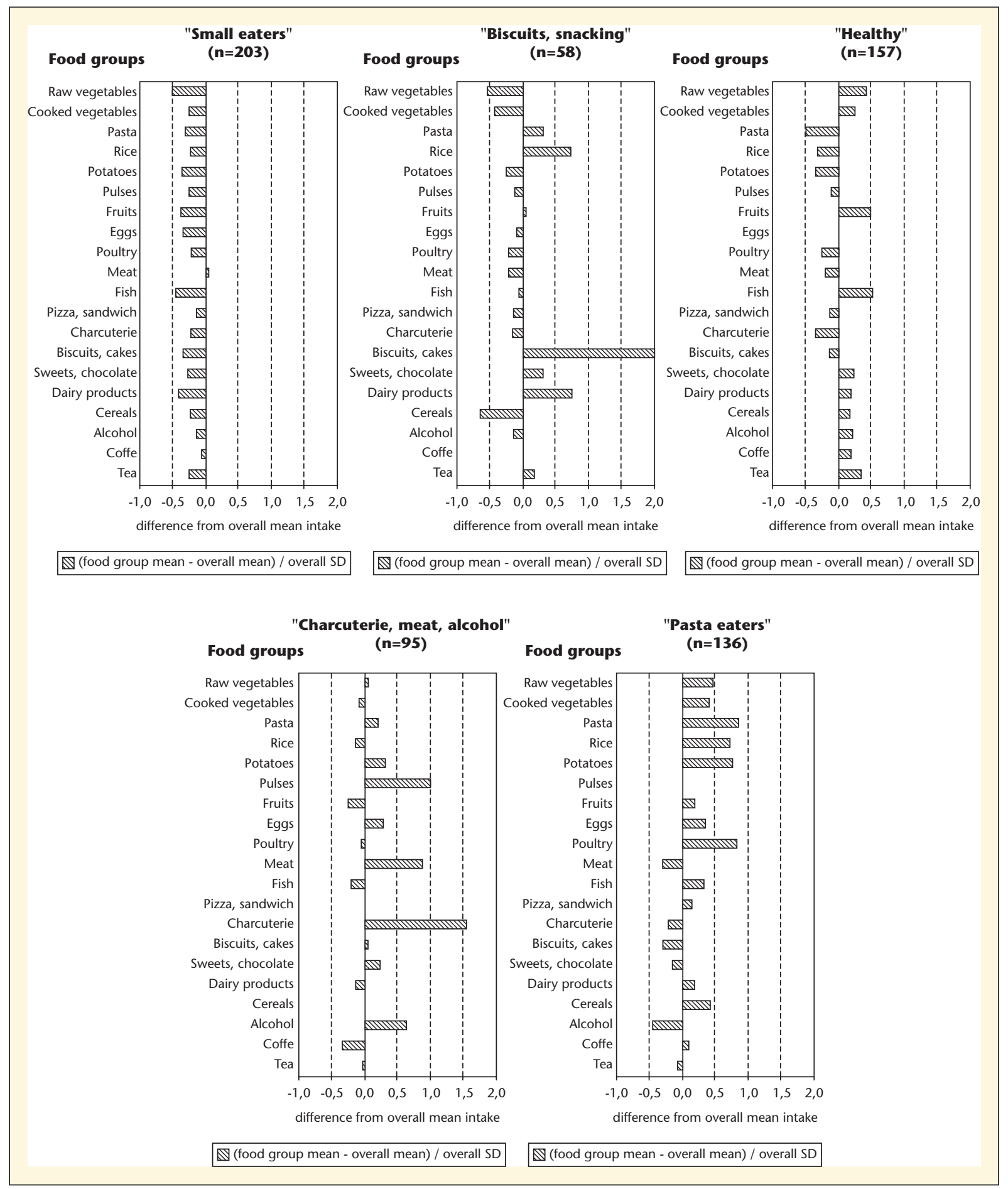

Figure 1. Dietary patterns among elderly men living in Bordeaux, France, the Three-City study, 2001-2002 (N=647), published in (Samieri et al., 2008).

account depressive status as a potential confounder (Samieri et al., 2008). Among the 1,214 subjects from the Bordeaux sample of the $3 C$ study who had plasma fatty acid measurement and were non-demented at baseline, 65 dementia cases were diagnosed over 4 y. Baseline plasma EPA and DHA were lower in future dementia cases compared to controls (mean plasma EPA proportion in future demented $=0.83 \%$ vs. $0.83 \%$ in controls, $P=0.03$; mean plasma DHA proportion $=2.20 \%$ in future demented vs. $2.41 \%$ in controls, $P=0.04)$. Each increase of 1 standard deviation (SD) $(=0.5 \%$, corresponding to approximately 0.5 weekly portion of fish) of plasma EPA at baseline was 


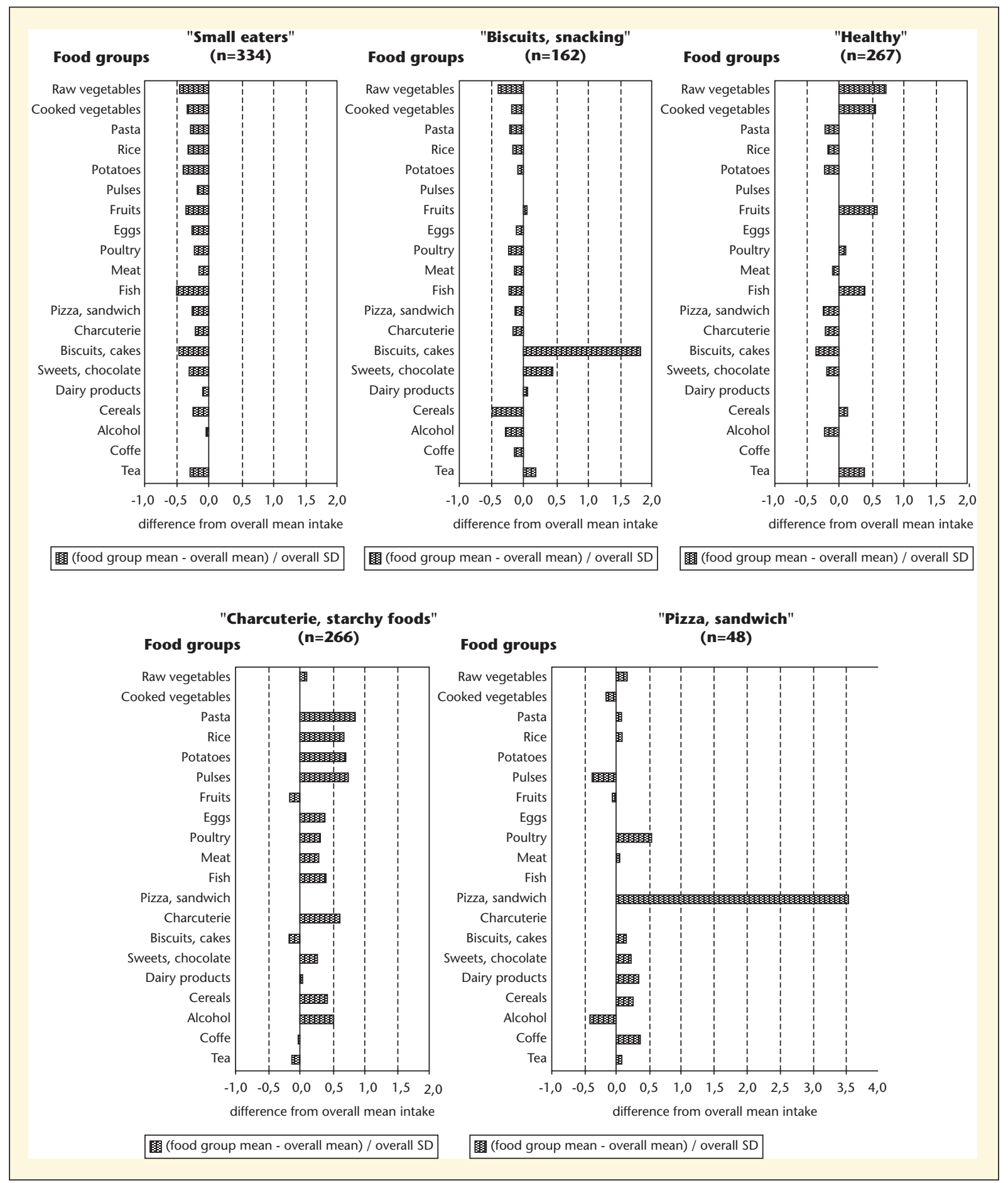

Figure 2. Dietary patterns among elderly women living in Bordeaux, France, the Three-City study, 2001-2002 (N=1,077), published in (Samieri et al., 2008).

associated with a $30 \%$ lower dementia risk after adjustment for age, gender, education, ApoE4 allele, diabetes, plasma triglycerides and plasma vitamin $\mathrm{E}$ (table 1). Further adjustment for baseline depressive status as assessed by CESD did not change the magnitude of this association, suggesting that depressive status and EPA status were independent predictors of dementia risk. In contrast, plasma DHA was not associated with dementia risk in multivariate models (table 1). This result suggested a specific preventive role for EPA in dementia which may have been 
Table 1. Associations between plasma EPA and DHA proportions and 4-y incident dementia in the Bordeaux sample of the Three-City study (N=1,214), 1999-2004, published in (Samieri et al., 2008)

\begin{tabular}{|c|c|c|c|c|c|c|}
\hline \multirow[b]{3}{*}{ Plasma fatty acids } & \multicolumn{6}{|c|}{ Risk of dementia } \\
\hline & \multicolumn{3}{|c|}{ Model $1^{1}$} & \multicolumn{3}{|c|}{ Model $\mathbf{2}^{2}$} \\
\hline & HR & $95 \% \mathrm{CI}$ & $\boldsymbol{P}$ & HR & $95 \% \mathrm{Cl}$ & $\mathbf{P}$ \\
\hline \multicolumn{7}{|l|}{ EPA } \\
\hline for 1 SD of plasma proportion & 0.69 & $0.48,0.98$ & 0.04 & 0.69 & $0.48,0.98$ & 0.04 \\
\hline Depressive status & - & - & - & 2.16 & $1.08,4.34$ & 0.03 \\
\hline \multicolumn{7}{|l|}{$\mathrm{DHA}$} \\
\hline for 1 SD of plasma proportion & 0.84 & $0.63,1.11$ & 0.21 & 0.83 & $0.62,1.10$ & 0.19 \\
\hline Depressive status & - & - & - & 2.22 & $1.10,4.46$ & 0.03 \\
\hline
\end{tabular}

${ }^{1}$ Cox proportional hazard models with delayed entry taking age as a time scale and adjusted for education (in four levels), ApoE4 allele, diabetes, plasma triacylglycerides, plasma vitamin E.

${ }^{2}$ Model $1+$ depressive status.

Abbreviations: EPA: eicosapentaenoic acid; DHA: docosahexaenoic acid; HR: Hazard Ratio; SD: Standard Deviation.

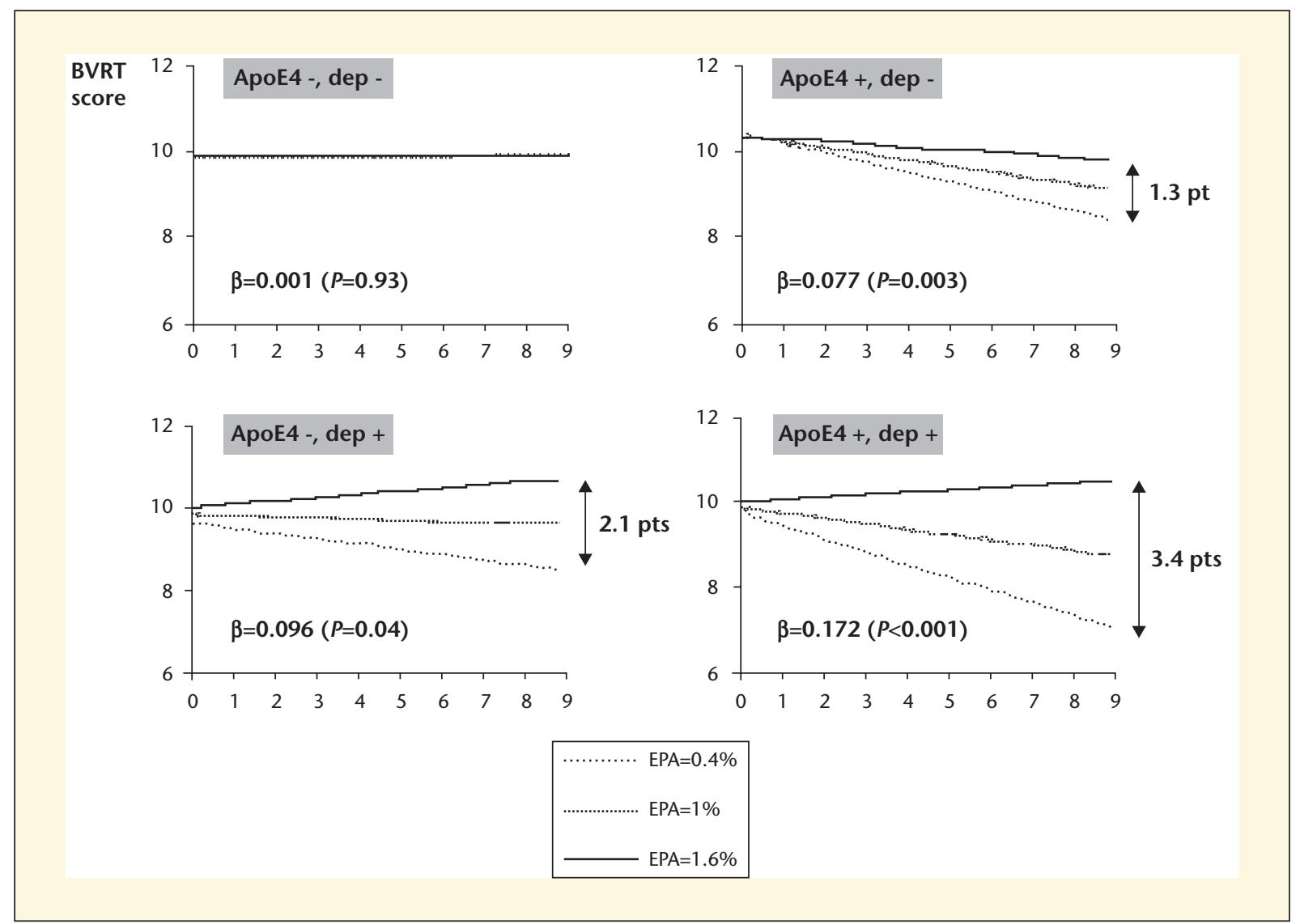

Figure 3. Evolution of predicted mean BVRT score for three typical levels of plasma EPA proportion according to depressive symptoms and ApoE4 carrier status, estimated in a multivariate linear mixed model in participants from the Bordeaux Sample of the Three-City Study non demented at baseline (1999-2006) ( $N=1,130)$, published in (Samieri, Feart et al., 2010). ApoE4 carriers (at least one - 44 allele) were noted as ApoE4 + and ApoE4 non carriers as ApoE4. Participants with high depressive symptoms were noted as dep + and those with low depressive symptoms as dep-.

Predicted mean evolution of cognitive scores over time were represented for men with average age (74 y), body mass index $\left(26.4 \mathrm{~kg} / \mathrm{m}^{2}\right)$, number of drugs regularly consumed (4), plasma vitamin $E(15.8 \mathrm{mmol} / \mathrm{L})$ and triglycerides $(1.3 \mathrm{mmol} / \mathrm{L})$, and who had a low level of education, were married, non-smokers, who did not consume alcohol or use antidepressants, who had no history of cardiovascular disease, no diabetes and no recent weight loss $>3 \mathrm{~kg}$. The three typical values of plasma EPA were chosen as: overall mean - 1 SD; overall mean; overall mean + 1 SD.

In each group, Beta coefficients and their p-value were given for EPA $\times$ Time term (corresponding to the variation in slope associated with each increase of 1 standard deviation of plasma EPA).

Abbreviations: BVRT: Benton Visual Retention Test; EPA: eicosapentaenoic acid. 
underestimated in previous studies, possibly involving anti-inflammatory, vascular or energy metabolic pathways (Freeman et al., 2006; Freemantle et al., 2006).

\section{Plasma long-chain n-3 PUFA and cognitive decline}

Secondly, the associations between plasma EPA and DHA and cognitive decline to several cognitive domains were examined, taking into account ApoEs4 allele and depression as potential modifying factors (Samieri et al., 2010). The study included 1,228 subjects from the Bordeaux sample of the $3 C$ study with plasma fatty acid measurements who were non-demented at baseline and who had been evaluated at least once for cognition over $7 \mathrm{y}$ of follow-up. Baseline plasma EPA, but not DHA, was lower in subjects with high depressive symptoms (mean plasma EPA in subjects with high depressive symptoms $=0.89 \% \mathrm{Vs}$. $1.02 \%$ in those with low depressive symptoms, $P<0.05$; mean plasma DHA in subjects with high depressive symptoms $=2.40 \%$ vs . $2.39 \%$ in those with low depressive symptoms).

When examining the association between plasma long-chain n-3 PUFA and the evolution of cognitive performances, a modifying effect of ApoE4 status and depressive status was found.

Each increase of 1 SD of plasma EPA at baseline was associated with an attenuation of decline to working memory performances as assessed by BVRT, particularly in those with depressive symptoms, and in those carrying the ApoE4 allele. In those both depressed and carrying ApoE4, the increase of plasma EPA from $0.4 \%$ to $1.6 \%$ lead to a 3.4 points difference in BVRT scores expected over 9 y (figure 3). Plasma DHA was also associated with lower decline to working memory in ApoE4 carriers. In this sample, EPA and DHA were not associated with the evolution of performances in the other cognitive domains (verbal fluency as assessed by IST, global cognition as assessed by MMSE, executive functions as assessed by TMT).

This latter study therefore suggests that EPA and DHA may contribute to delaying decline in visual working memory in ApoE4 carriers, suggesting a nutrient- gene interaction which deserves further research. In older depressed subjects, EPA, but not DHA, may slow down cognitive decline, possibly through an anti-depressant effect.

\section{Conclusion}

Overall, the results from this thesis suggest that cognitive performances and depressive symptoms, which are two major features of brain aging, may vary according to habitual dietary habits of subjects, and may be improved in higher fish and fruits and vegetables consumers. Moreover, we brought some evidence that higher adherence to the MeDi may contribute to slowing down age-related global cognitive decline. While one of the key nutrients for the prevention of cognitive decline may be long-chain n-3 PUFA, our results emphasize the role of EPA. We showed that increase of 1 SD of plasma EPA (corresponding to increase of 0.5 fish weekly servings) was associated with a 30\%-decreased dementia risk over $4 \mathrm{y}$. We also evidenced new and puzzling interactions with the ApoE4 genotype and depressive symptomatology on cognitive decline that will deserve further research.

Taken altogether, these results suggest that slowing down cognitive decline could be achieved by healthy dietary habits, maybe more efficiently in some individuals targeted according to their genetic and psychosocial characteristics. Nevertheless, randomized controlled trials are needed to demonstrate the impact of shifting to healthy diets on trajectories of cognitive decline.

\section{REFERENCES}

American Psychiatric Association. Diagnostic and Statistical Manual of Mental Disorders (IV). Washington DC, American Psychiatric Press, 1994.

American Psychiatric Association. Diagnostic and Statistical Manual of Mental Disorders (IV-TR). 4th Text Revised. Washington DC, American Psychiatric Press, 2000.

Amieva H, Le Goff $\mathrm{M}$, et al. Prodromal Alzheimer's disease: successive emergence of the clinical symptoms. Ann Neurol 2008; 64: 492-8.

Brayne $\mathrm{C}$. The elephant in the room - healthy brains in later life, epidemiology and public health. Nat Rev Neurosci 2007; 8: 233-9.
Cunnane SC, Plourde M, et al. Fish, docosahexaenoic acid and Alzheimer's disease. Prog Lipid Res 2009; 48: 239-56.

Dubois $\mathrm{B}$, Feldman $\mathrm{HH}$, et al. Revising the definition of Alzheimer's disease: a new lexicon. Lancet Neurol 2010; 9: 1118-27.

Dubois B, Feldman HH, et al. Research criteria for the diagnosis of Alzheimer's disease: revising the NINCDS-ADRDA criteria. Lancet Neurol 2007; 6: 734-46.

Farrer LA, Cupples LA, et al. Effects of age, sex, and ethnicity on the association between apolipoprotein E genotype and Alzheimer disease. A meta-analysis. APOE and Alzheimer Disease Meta Analysis Consortium. Jama 1997; 278: 1349-56.

Feart C, Peuchant E, et al. Plasma eicosapentaenoic acid is inversely associated with severity of depressive symptomatology in the elderly: data from the Bordeaux sample of the Three-City Study. Am J Clin Nutr 2008; 87: 1156-62.

Feart C, Samieri C, et al. Diet and Alzheimer's disease: new evidence from epidemiological studies. In: New research on Nutrition in the prevention of Alzheimer's disease. Ramassamy\&Bastianetto, Research Signpost/Transworld Research Network, Trivandrum, 2010.

Feart C, Samieri C, et al. Adherence to a Mediterranean diet, cognitive decline, and risk of dementia. Jama 2009; 302: 638-48.

Feart C, Torres MJ, et al. Adherence to a Mediterranean diet and plasma fatty acids: data from the Bordeaux sample of the ThreeCity study. Br / Nutr 2011; 1-10.

Fotuhi M, Hachinski V, et al. Changing perspectives regarding late-life dementia. Nat Rev Neurol 2009; 5: 649-58.

Freeman MP, Hibbeln JR, et al. Omega-3 fatty acids: evidence basis for treatment and future research in psychiatry. / Clin Psychiatry 2006; 67: 1954-67.

Freemantle $\mathrm{E}$, Vandal $\mathrm{M}$, et al. Omega-3 fatty acids, energy substrates, and brain function during aging. Prostaglandins Leukot Essent Fatty Acids 2006; 75: 213-20.

Jack Jr CR, Knopman DS, et al. Hypothetical model of dynamic biomarkers of the Alzheimer's pathological cascade. Lancet Neurol 2010; 9: 119-28.

Lin PY, Huang SY, et al. A meta-analytic review of polyunsaturated fatty acid compositions in patients with depression. Biol Psychiatry 2010; 68: 140-7.

Moeller SM, Reedy J, et al. Dietary patterns: challenges and opportunities in dietary patterns research an Experimental Biology workshop, April 1, 2006. I Am Diet Assoc 2007; 107: 1233-9. 
Panza F, Frisardi V, et al. Late-life depression, mild cognitive impairment, and dementia: possible continuum? Am / Geriatr Psychiatry 2010; 18: 98-116.

Prince M, Jackson JC. World Alzheimer Report. London, England: A. s. D. International. 2009.

Reiman EM, Chen K, et al. Functional brain abnormalities in young adults at genetic risk for late-onset Alzheimer's dementia. Proc Natl Acad Sci U S A 2004; 101: 284-9.

Reitz C, Brayne C, et al. Epidemiology of Alzheimer disease. Nat Rev Neurol 2011; 7: 137-52.

Samieri C, Feart C, et al. Low plasma eicosapentaenoic acid and depressive symp- tomatology are independant predictors of dementia risk. Am J Clin Nutr 2008; 88: 714-21.

Samieri C, Feart C, et al. Omega-3 fatty acids and cognitive decline: modulation by ApoEepsilon4 allele and depression. Neurobiol Aging 2010. In press. doi:10.1016/j. neurobiolaging.2010.03.020

Samieri C, Jutand MA, et al. Dietary patterns derived by hybrid clustering method in older people: association with cognition, mood, and self-rated health. J Am Diet Assoc 2008; 108: 1461-71.

Sleegers K, Lambert JC, et al. The pursuit of susceptibility genes for Alzheimer's disease: progress and prospects. Trends Genet 2010; 26: 84-93.

Sofi F, Abbate R, et al. Accruing evidence about benefits of adherence to the Mediterranean diet on health: an updated systematic review and meta-analysis. Am / Clin Nutr 2010; 92: 1189-96.

Three-City study group. Vascular Risk factors and risk of dementia : design of the ThreeCity Study and baseline characteristics of the study population. Neuroepidemiology 2003; 22: 316-25.

Trichopoulou A, Kouris-Blazos A, et al. Diet and overall survival in elderly people. $B M$ J 1995; 311: 1457-60. 